\title{
AMACIAMENTO ESTÁTICO DO AÇO INOXIDÁVEL AUSTENITICO ALTO NITROGÊNIO ASTM F-1586*
}

\author{
Sebastião Raimundo de Jesus Belém Leitão Filho ${ }^{1}$ \\ Ramaiany Carneiro Mesquita ${ }^{2}$ \\ Bruno Leonardy Lopes ${ }^{3}$ \\ Gedeon Silva Reis ${ }^{4}$ \\ Eden Santos Silva ${ }^{5}$ \\ Cíntia Leite Rodrigues ${ }^{6}$
}

Resumo

A simulação física é uma alternativa no estudo dos eventos durante o processamento termomecânico, sem necessidade de equipamentos industriais, permitindo a realização de projetos de deformações similares as de escala industrial, porém em diferentes condições de atrito e transferência de calor. O objetivo desta pesquisa foi investigar o retardo na cinética de amaciamento estático no aço inoxidável austenitico ASTM F-1586, usado na confecção de implantes ortopédicos. A metodologia aplicada consistiu no cálculo da fração de amaciamento $\left(\mathrm{X}_{\mathrm{s}}\right)$, após realização dos ensaios de compressão a quente com duplo passe de deformação num dilatômetro, nas temperaturas de $1000-1200{ }^{\circ} \mathrm{C}$, taxa de deformação de $1,0 \mathrm{~s}^{-1}$ e intervalos entre passes variando de 1 a $1000 \mathrm{~s}$. Os resultados indicaram que o aço ASTM F-1586 sofre recristalização metadinâmica em deformações maiores que 0,30 , onde as curvas $X_{s}$ vs. t para temperaturas abaixo de $1100^{\circ} \mathrm{C}$ apresentaram formação de patamares com atraso na cinética amaciamento, resultante da interação recristalização-precipitação (Fase Z) induzida por deformação no interpasse, sendo menos efetivo quanto maior a temperatura e a deformação.

Palavras-chave: Simulação física; Processamento termomecânico; Aço inoxidável; Recristalização metadinâmica e Precipitação.

\section{STATIC SOFTENING OF AUSTENIC STAINLESS STEEL N-HIGH ASTM F-1586}

\begin{abstract}
Physical simulation is an alternative in the study of events during thermomechanical processing, without the need of industrial equipment, allowing the accomplishment of strain projects, such as industrial scale, under different conditions of discharge and discharge of hot. The objective of this research was to investigate the delay in the kinetics of static softening in austenitic stainless steel ASTM F-1586, used in the manufacture of orthopedic implants. The applied methodology consisted of the calculation of the softening fraction $\left(\mathrm{X}_{\mathrm{s}}\right)$, after the double fit compression tests in a dilatometer, at temperatures of $1000-1200{ }^{\circ} \mathrm{C}$, strain rate of $1.0 \mathrm{~s}^{-1}$ and intervals between passes varying from 1 to $1000 \mathrm{~s}$. The results indicated that ASTM F-1586 steel undergoes metadynamic recrystallization (MDRX) in strains greater than 0.30, whereas $X_{s}$ vs. $t_{p}$ for temperatures below $1100{ }^{\circ} \mathrm{C}$ show formation of delayed in the softening kinetic, resulting from the recrystallization-precipitation interaction (Z-Phase) strain-induced by interpass time, the less effective the higher the temperature and the strain.
\end{abstract}

Keywords: Physical simulation; Thermomechanical processing; Stainless steel; Metadynamic recrystallization and precipitation.

1 Engenharia Mecânica/Engenharia Mecânica, Mestrando, Aluno/Aluno, Programa de Pós Graduação em Engenharia dos Materiais/Engenharia Mecânica, Instituto Federal de Ciência e Educação do Maranhão (IFMA), São Luís, Maranhão, Brasil. 
2 Física/Física, Mestre, Professora/Professora, Engenharia de Materiais/ Engenharia de Petróleo, Universidade CEUMA, São Luís, Maranhão, Brasil.

3 Engenharia Mecânica/Engenharia Mecânica, Mestre, Professor/Professor, Engenharia Mecânica/Engenharia Mecânica, Instituto Federal de Ciência e Educação do Maranhão (IFMA), São Luís, Maranhão, Brasil.

4 Engenharia Mecânica/Engenharia Mecânica, Doutor, Professor/Professor, Engenharia Mecânica/Engenharia Mecânica, Instituto Federal de Ciência e Educação do Maranhão (IFMA), São Luís, Maranhão, Brasil.

5 Física/Física, Doutor, Professor/Professor, Engenharia e Ciência dos Materiais/ Engenharia Mecânica, Universidade CEUMA, São Luís, Maranhão, Brasil.

6 Engenharia Químical Engenharia Química, Mestre, Professora/Professora, Engenharia de Petróleo/Engenharia de Petróleo, Universidade CEUMA, São Luís, Maranhão, Brasil. 


\section{INTRODUÇÃO}

Aperfeiçoar o processamento dos materiais metálicos é um dos principais focos da indústria metalúrgica devido à necessidade de materiais mais resistentes e produtos com menor peso, além de atender as especificações mais rigorosas impostas por consumidores e normas reguladoras. Assim a produção de aços com elevada resistência mecânica sem grandes adições de elementos de ligas, em especial os de custo mais elevados, só se tornou possível com o entendimento da metalurgia física do processamento industrial [1,2]. Neste ramo existem diversos parâmetros complexos, tais como tempo, deformação, taxa de deformação e efeito da temperatura que se relacionam entre si. Por outro lado, a combinação com fenômenos metalúrgicos mecânicos e microestruturais provocam mudanças significativas nas características da liga, como a tensão de escoamento, tamanho de grão, influenciando na forma do processo.

Durante 0 processamento de ligas metálicas deformadas a quente (laminação a quente, forjamento e extrusão) não se pode eliminar completamente o encruamento, pois resultaria na instabilidade da microestrutura [3,4]. Dessa forma após o processamento de deformação a quente no intervalo de tempo entre passes, a microestrutura continua a se desenvolver, com alivio do encruamento e estabilidade do estado estacionário e reorganização microestrutural no material, com predominância dos mecanismos de amaciamento: recuperação estática (SRV), recristalização estática (SRX) e recristalização metadinâmica (MDRX), sendo que as propriedades dos componentes metálicos são fortemente dependentes da rota e dos parâmetros do processamento industrial.

Um a forma de se estudar o comportamento mecânico e microestrutural de ligas metálicas especiais em linha industrial são por meio de simulações físicas em escala laboratorial. Amostras são submetidas a ciclos térmicos e mecanismos similares as que são aplicadas nos processos, com réplica de toda seqüência de passes de deformação com imposição dos parâmetros do processamento industrial na confecção próteses ortopédicas com geometria especifica [5,6]. Em seguida o comportamento plástico dos mecanismos de endurecimento e amaciamento do material é parametrizado por meio de equações constitutivas e evolutivas das ligas segundo suas condições de deformação.

Dentro da classe de aços inoxidáveis, os que apresentam alto teor $\mathrm{N}$ têm sido usados em aplicações biomédicas, o aço ASTM F-1586 que representa uma liga com baixo $\mathrm{C}, \mathrm{Mn}, \mathrm{Ni}$, $\mathrm{Nb}$ apresenta um balanço entre resistência mecânica e resistência a corrosão com grande aplicação na confecção de próteses ortopédicas (Cabeça femoral, articulação superior de joelho e placa articulação mandibular) [7]. Algumas pesquisas na literatura têm reportado o comportamento termomecânico da série do aço AISI F-1586 (ISO 5832-9) quanto aos fenômenos metalúrgicos sob deformação a quente na obtenção de limite de resistência considerável, resistência a corrosão geral e localizada satisfatória e biocompatibilidade [1,7].

Neste trabalho será investigado o comportamento da cinética de recristalização estática sob teste de compressão a quente de duplo passe de deformação a quente isotérmico, investigando a possível interação dos mecânicos de amaciamento estático e os mecanismos de resistência da liga, com intuito de melhor sua trabalhabilidade e otimização dos parâmetros de processamento a quente na planta industrial. Para mensurar o amaciamento entre passes será a plicado o "Método $0,2 \%$ offset", cuja representação esquemática é ilustrado na Figura $1[8,9]$. O Método offset é baseado na seguinte relação: 


$$
X_{s}=\frac{\sigma_{m}-\sigma_{y}}{\sigma_{m}-\sigma_{o}}
$$

Onde, $\sigma_{\mathrm{m}}$ é a tensão de escoamento máxima, $\sigma_{\mathrm{y}}$ é a tensão de escoamento no segundo passe; $\sigma_{\circ}(0,2 \%)$ é a tensão de início de escoamento plástico.

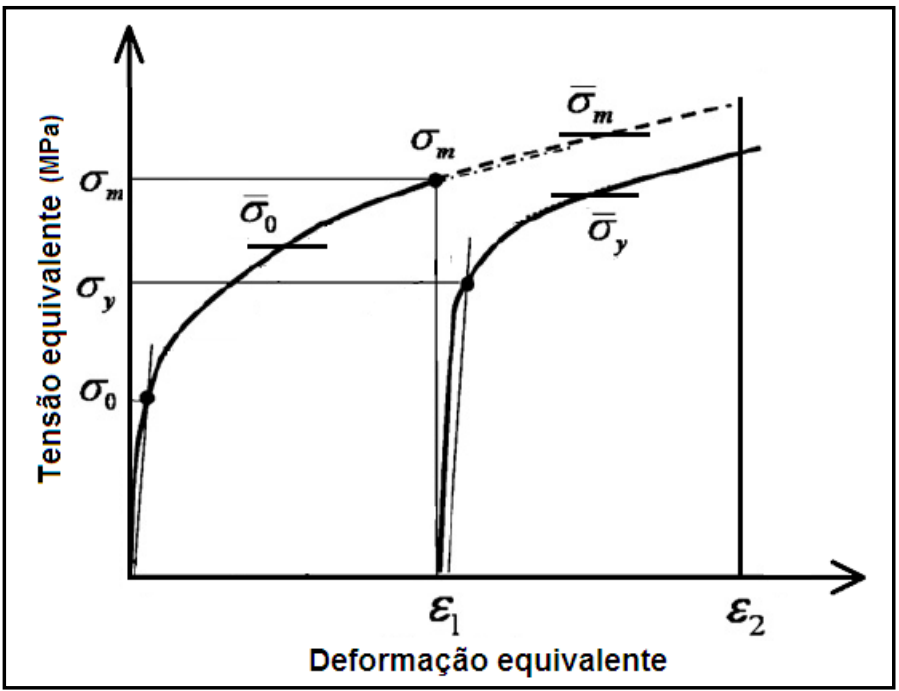

Figura 1 Esquema indicando os valores de tensões utilizados para o cálculo da fração amaciada. Métodos offset e tensão média equivalente.

\section{MATERIAIS E MÉTODOS}

O aço AISI F-1586 foi investigado neste trabalho, cuja composição química está listada na Tabela 1. Os corpos de prova cilíndricos com dimensões $\Phi 5 \times 10 \mathrm{~mm}$ (soldados em suas extremidades com finos discos de Mo, 0,06 mm para redução de atrito) foram aquecidos por indução a uma taxa de aquecimento de $5,5{ }^{\circ} \mathrm{C} / \mathrm{s}$ até uma temperatura de $1250{ }^{\circ} \mathrm{C}$ e mantido por $300 \mathrm{~s}$ para solubilização, em seguida submetidos a teste de compressão a quente de duplo passe de deformação conduzido num Dillatômetro, modelo DIL 850 A/D, Bahr Thermoanalyse Figura 2, nas condições de $1000,1050,1100,115^{\circ}$ e $1200^{\circ} \mathrm{C}$, com aplicação de deformação $(0,15$ e 0,30$)$ a uma taxa de deformação de $0,1 \mathrm{~s}^{-1}$, com tempo entre passe com interrupção entre as duas deformações variou de 1 a 1000s. A partir das curvas $\sigma$ vs, $\varepsilon$ experimentais calculou-se a fração amaciada $\left(X_{s}\right)$ entre passe de deformação para diferentes temperaturas e tempos de espera $\left(t_{p}\right)$ usando o "Método offset $0,2 \%$ " $[9,10]$.

Tabela 1 Composição química do aço ASTM F-1586, (\%massa).

\begin{tabular}{cccccccccccc}
\hline \hline $\mathbf{C}$ & $\mathbf{S i}$ & $\mathbf{M n}$ & $\mathbf{N i}$ & $\mathbf{C r}$ & $\mathbf{M o}$ & $\mathbf{S}$ & $\mathbf{P}$ & $\mathbf{N}$ & $\mathbf{C u}$ & $\mathbf{N b}$ & $\mathbf{F e}$ \\
\hline 0,035 & 0,37 & 4,04 & 10,6 & 20,3 & 2,47 & 0,001 & 0,022 & 0,36 & 0,06 & 0,29 & bal. \\
\hline \hline
\end{tabular}




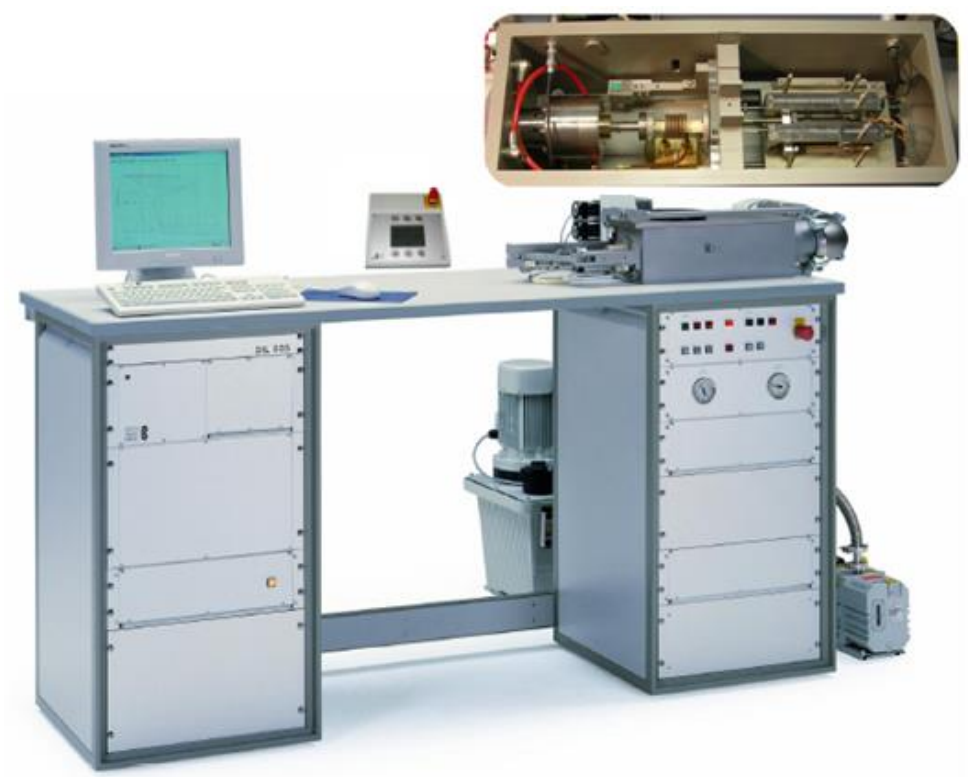

Figura 2 Dilatômetro modelo DIL 850 A/D Bahr Thermoanalyse usado nos ensaios de compressão a quente com de duplo passe de deformação.

Para investigar o comportamento microestrutural durante a deformação a quente de duplo passe foram realizados alguns ensaios de compressão a quente interrompidos, seguida de resfriamento brusco em água. A ocorrência ou não da recristalização metadinâmica foi investigada utilizando as seqüências de micrografias em diferentes condições de deformação. A microestrutura foi analisada por microscopia eletrônica de varredura, modelo Phillips XL30 FEG.

\section{RESULTADOS E DISCUSSÃO}

\subsection{Curvas $\sigma$ vs. $\varepsilon$ isotérmico contínuo}

A Figura 3 apresenta as curvas de escoamento plástico nas condições de compressão a quente isotérmico continuo, evidenciando diferentes níveis de resistência do aço com diferentes temperatura de deformação aplicada, evidenciando que a recristalização dinâmica se inicia a níveis de deformações abaixo de 0,30 , onde a deformação de pico $\left(\varepsilon_{p}\right)$ apresenta valores maiores calculados analiticamente.

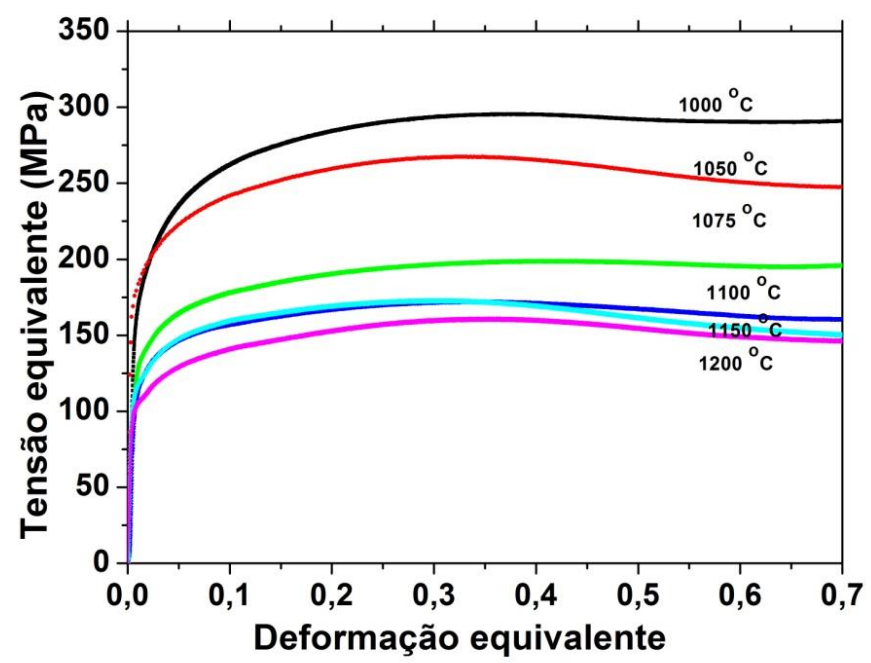

Figura 3 Curva de escoamento plástico dos testes de compressão a quente isotérmico contínuo. 
As curvas de escoamento plástico mostradas na Figura 3 têm a forma típica apresentada pelos materiais metálicos que recristalizam dinamicamente quando deformados a quente. Inicialmente há uma região de encruamento, na qual a tensão de escoamento plástico aumenta continuamente, apresentando uma alteração na curvatura conforme o material é deformado. Com o prosseguimento da deformação alcança-se um máximo correspondente ao pico de tensões. Por fim, há uma etapa de amaciamento por deformação com redução no nível de tensão para um valor intermediário, podendo ou não alcançar a tensão de estado estacionário.

As curvas de escoamento plástico isotérmico contínuo dos ensaios de compressão a quente apresentaram picos de tensão, característicos de recristalização dinâmica (DRX) durante a deformação aplicada, Fig 3. Após o pico de tensão $\left(\sigma_{p}\right)$, o nível de tensão cai levemente até o estado estacionário $\left(\sigma_{\mathrm{ss}}\right)$, com amaciamento dinâmico cadenciado nas condições de baixa temperatura e taxa de deformação intermediária.

\subsection{Curvas $\sigma$ vs. $\varepsilon$ duplo passe}

As curvas de escoamento plásticas obtidas com os testes de compressão a quente com duplo passe de deformação são apresentadas nas Figura 4. Vê-se que durante o primeiro passe de deformação $\left(\varepsilon_{1}\right)$ o material apresenta um aumento contínuo no nível de tensão com a deformação. O nível de tensão do segundo passe, com tempo de espera de $1,0 \mathrm{~s}$, aumenta rapidamente até o nível da curva do primeiro passe, principalmente para deformação menor que 0,30 não havendo amaciamento significativo e a curva do segundo passe coincide com a extrapolação da primeira. Entretanto, quando o tempo entre passe $\left(t_{p}\right)$ é maior que $10 \mathrm{~s}$, o amaciamento ocorre significativamente e a segunda curva apresenta níveis de tensão compatíveis com ao início do carregamento e o amaciamento é parcial. Quando o tempo entre passe é suficientemente longo o amaciamento ocorre completamente, e a curva do segundo passe é similar ao da primeira.
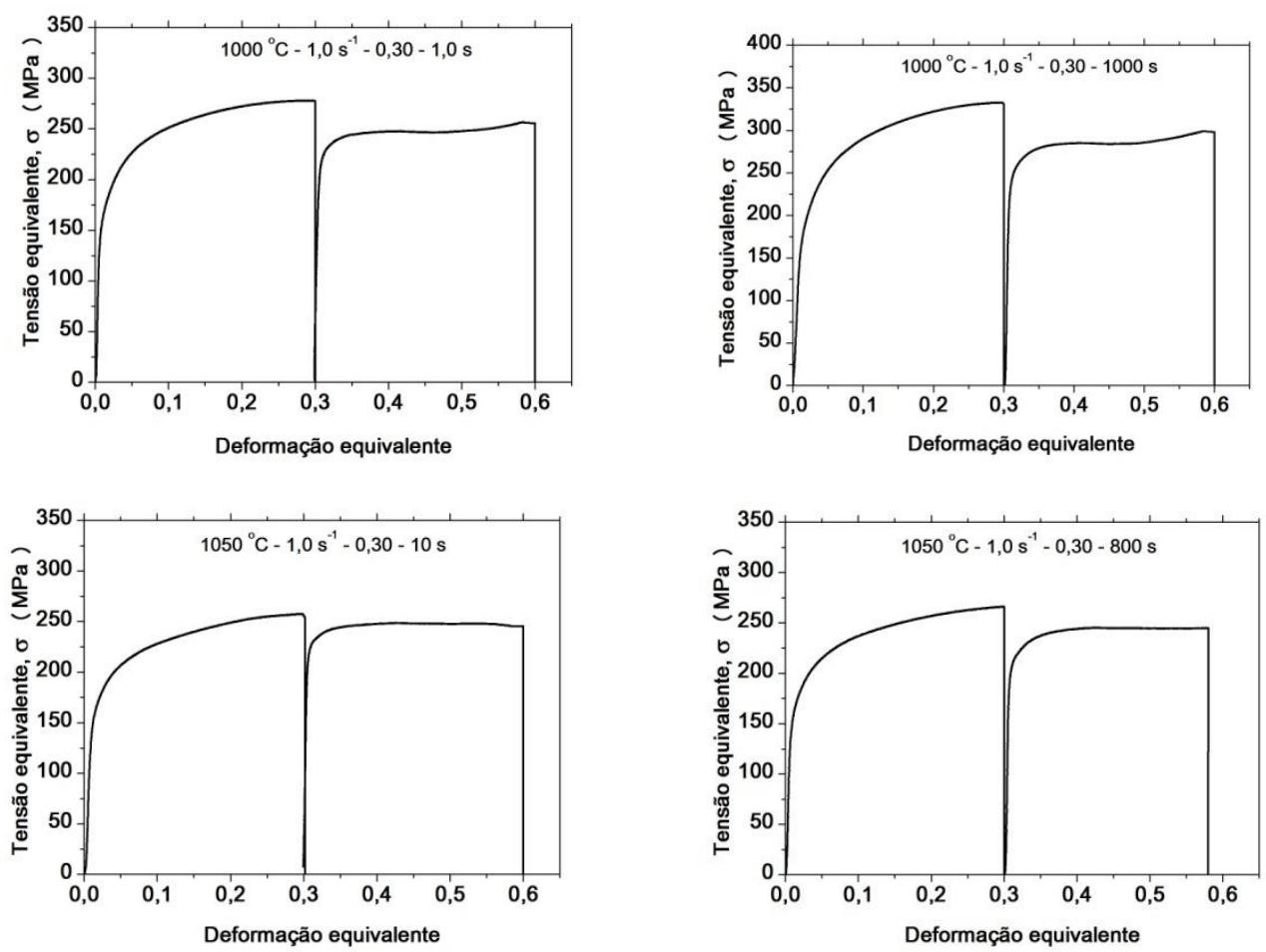

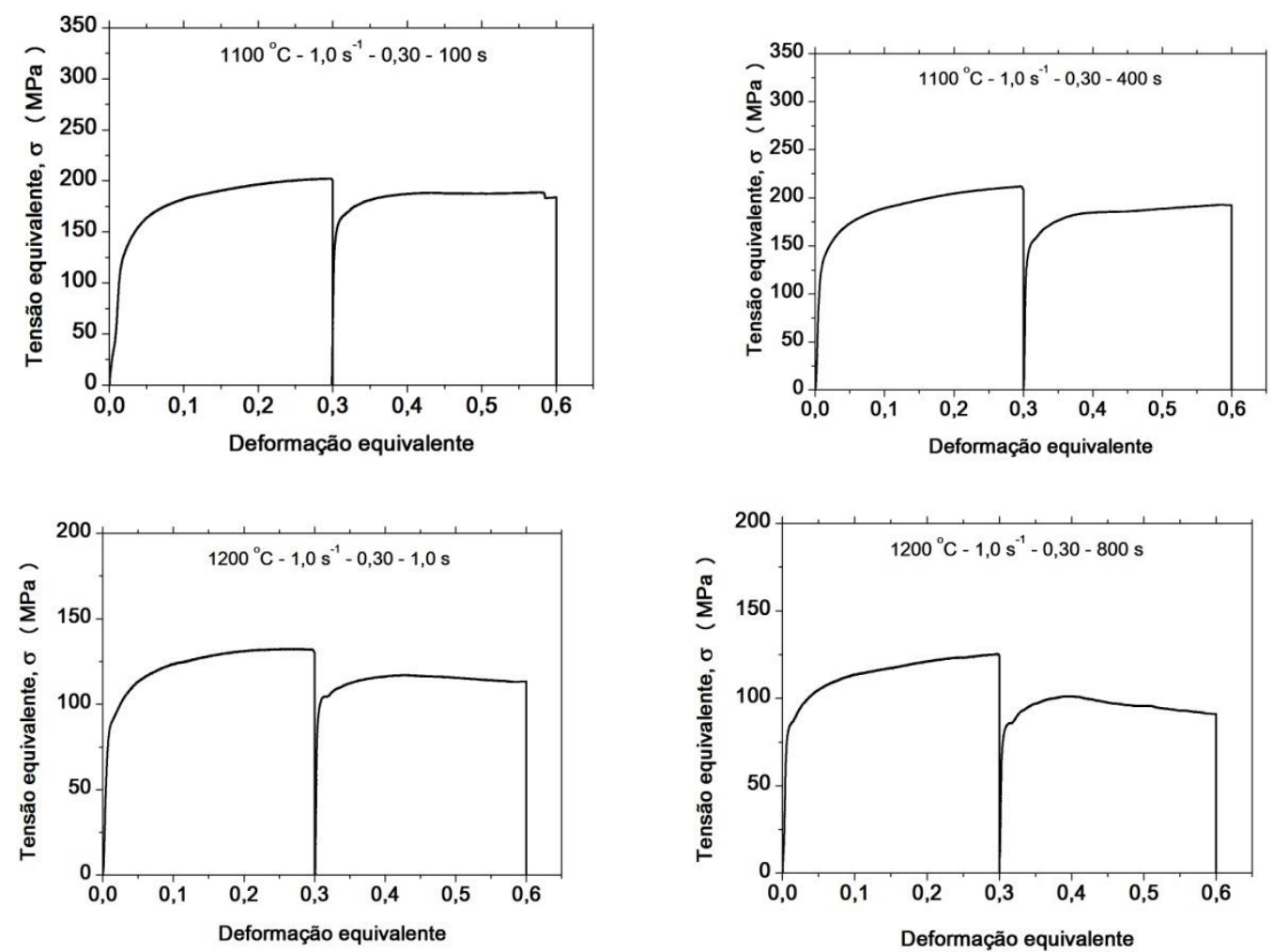

Figura 4 Curvas de escoamento plástico com duas deformações de 0,3 a $1000-1200{ }^{\circ} \mathrm{C}$ com taxa de deformação de $1,0 \mathrm{~s}^{-1}$ e tempo de espera variando de 1 a $1000 \mathrm{~s}$.

De forma geral, as curvas de amaciamento apresentam dois comportamentos diferenciados segundo as condições de deformação, cuja transição ocorre próxima a temperatura de $1100^{\circ} \mathrm{C}$, Fig. 4. Para temperaturas maiores as curvas apresentam a forma sigmoidal com amaciamento completo antes de iniciar a precipitação [11-13]. Enquanto que em temperaturas menores que $1100^{\circ} \mathrm{C}$, em menor quantidade de deformação, tem-se a formação dos patamares que retardam o amaciamento no interpasse. Vê-se que há aumento na taxa de amaciamento estático $\left(\mathrm{X}_{\mathrm{s}}\right)$ por recuperação estática $(\mathrm{SRV})$ com redução na temperatura de deformação, sendo responsável por quase $20 \%$ no início do tempo entre passe.

\subsection{Curvas da fração de amaciamento $\left(X_{s}\right)$ vs tempo entre passe ( $\left.t\right)$}

Os valores da fração amaciada (Xs) calculados pelo "Método offset $0,2 \%$ " estão apresentados na Figura 5, vê-se a evolução da fração de amaciamento $\left(X_{s}\right)$ em função do tempo entre passe ( $t_{p}$ ) para amostras deformadas com 0,15 e 0,30 a 1000 - $1200^{\circ} \mathrm{C}$. Verifica-se que a fração amaciada é sensível a variação na temperatura de deformação, havendo um amaciamento considerável para um intervalo entre passes de apenas $1,0 \mathrm{~s}$, seguida de um acréscimo na fração amaciada $\left(X_{\mathrm{s}}\right)$ com aumenta no tempo e na temperatura. Quanto maior a temperatura, menor o tempo necessário para que o material amacie completamente. Este fato leva a dois comportamentos

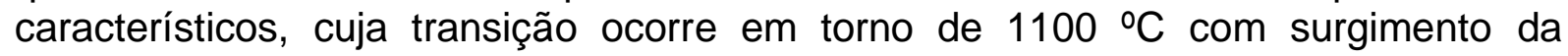
formação de patamares no nível de amaciamento. Por outro lado, à medida que a temperatura diminui a fração amaciada é reduzida com mudança na forma da curva. 


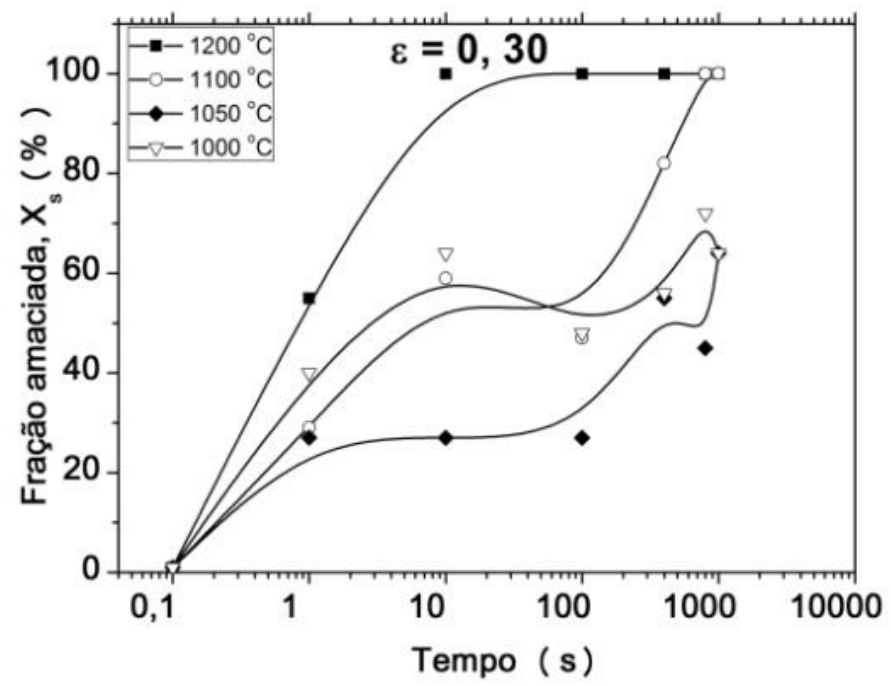

Figura 5 Fração amaciada $\left(X_{s}\right)$ em função do intervalo entre passe $\left(t_{p}\right)$ dos ensaios de duplo passe de compressão a quente: (a) $\varepsilon=0,30$.

Devido ao tempo de incubação para iniciar a recristalização estática (SRX), mais de $30 \%$ do amaciamento é devido à recuperação estática (SRV). À medida que o tempo entre passe $\left(t_{p}\right)$ aumenta a liga metálica não amacia completamente no interpasse, pois a fração amaciada $\left(X_{s}\right)$ se mantém em torno de $50 \%$ para tempos longos, mantendo a formação do patamar, Fig 5 . Esta condição está associada à formação de precipitados da fase $\mathrm{Z}(\mathrm{NbCrN})$ induzida por deformação que retarda a cinética de MDRX que se inicia com tempo entre passe de 1,0 s reduzindo a recuperação estática, inibindo a mobilidade dos contornos de grãos recristalizados estaticamente [14,15]. Para deformação menor, $\varepsilon=0,15$, essa interação ocorre em tempos maiores, aproximadamente $10 \mathrm{~s}$, sendo um fenômeno termicamente ativado.

\subsection{Estimativa do expoente de Avrami a partir das curvas $\left(X_{s}\right)$ vs $\left(t_{p}\right)$}

Para estimar o valor do expoente de Avrami (n) no tempo entre passe de deformação, aplica-se a linearização da fração de amaciamento $\left(X_{s}\right)$ e o tempo entre passe de deformação normalizado ( $\left.t / t_{0,5}\right)$, Figura 6 . Nota-se que o valor de $n$ é variável, $0,09<\mathrm{n}<0,23$, obtido por ajuste linear de múltiplas variáveis. Este valor do expoente de Avrami (n) é reportado na literatura a valores menores que 1,0 para aços em geral [12-14]. 


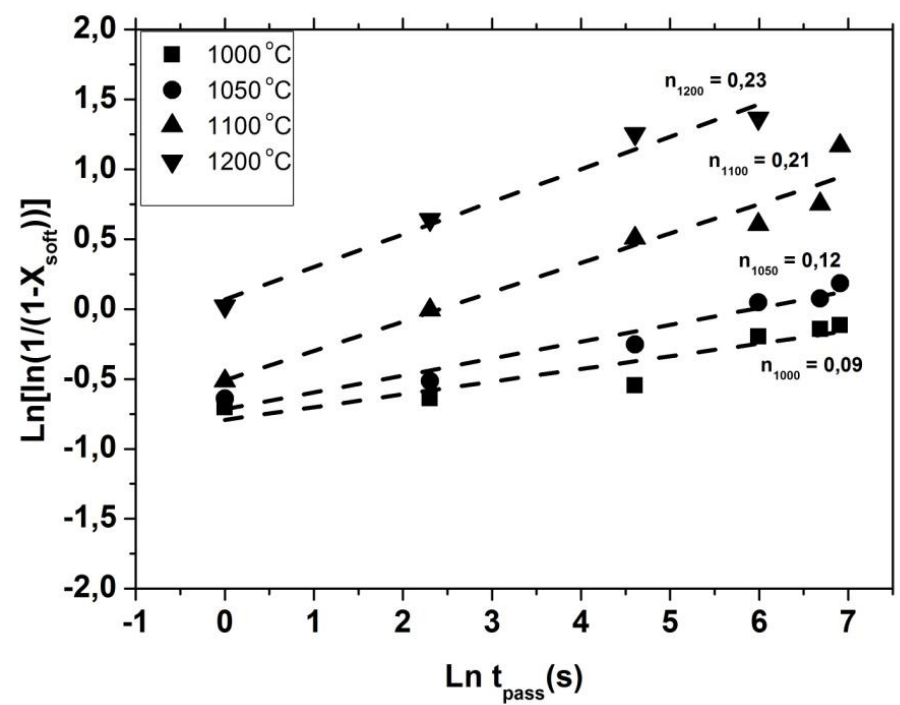

Figura 6 Determinação do expoente de Avrami (n) no amaciamento estático: $\varepsilon=0,3$ a $1000{ }^{\circ} \mathrm{C} c o m$ taxa de $1,0 \mathrm{~s}^{-1}$ e tempo entre passe entre 1 a $1000 \mathrm{~s}$.

O surgimento dos diferentes valores para o expoente de Avrami (n) deve à sua dependência com as condições de deformação (temperatura, deformação, tempo entre passe e taxa de deformação) e as respostas microestruturais do material com alta taxa de recuperação estática (SRV), atrasando a nucleação e crescimento dos grãos recristalizados metadinamicamente. Nota-se que há uma redução sutil no valor do expoente de Avrami (n) quando o tamanho de grão de grão diminui.

\subsection{Aspecto microestrutural nos amaciamento estático}

A microestrutura mostrada na Figura 7 evidencia a fração recristalizada metadinamicamente deformado a 0,30 na temperatura de $1050{ }^{\circ} \mathrm{C}$ com diferentes tempo entre passe $\left(t_{p}\right)$, com a cinética de MDRX apresentando baixo coeficiente de Avrami (n) mesmo sob alta temperatura de deformação a quente. Este valor baixo no coeficiente de Avrami (n) deve-se a recristalização metadinamicamente parcialmente, com forte evidencia de recuperação estática (SRV) e a presença de precipitados induzidos por deformação nos intervalos entre passe de deformação, Figura 8. 0 calculo da fração recristalizada por MEV/EBDS, mostrou que a orientação dos contornos de grãos mostrou que aproximadamente $60 \%$ são contornos de baixo ângulo, Figura 8, sendo uma constatação.
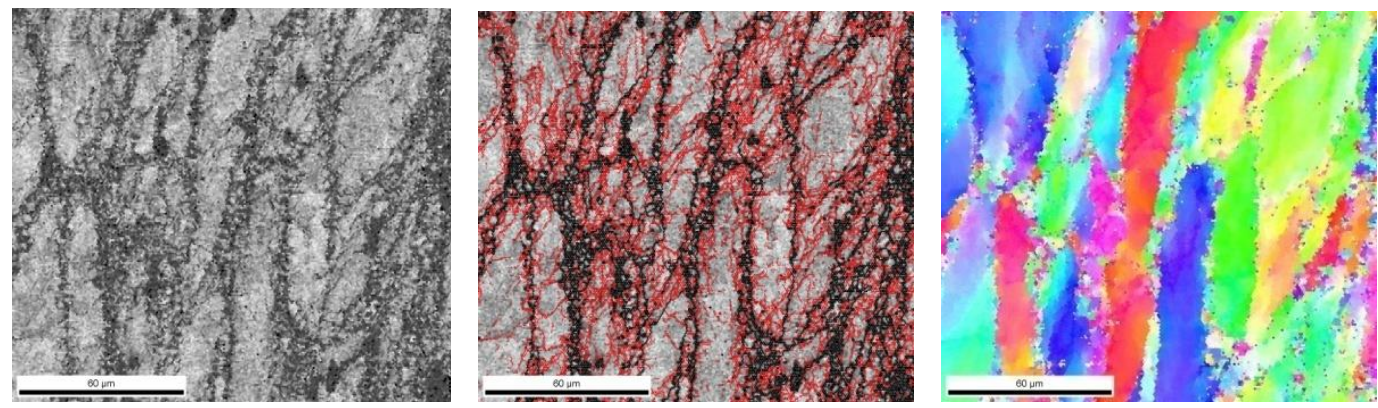

Figura 7 Cálculo da fração de recristalização metadinâmica por MEV/EBSD na temperatura de 1000 ${ }^{\circ} \mathrm{C}$, deformação de 0,30 e taxa de deformação de $1,0 \mathrm{~s}^{-1}$. 

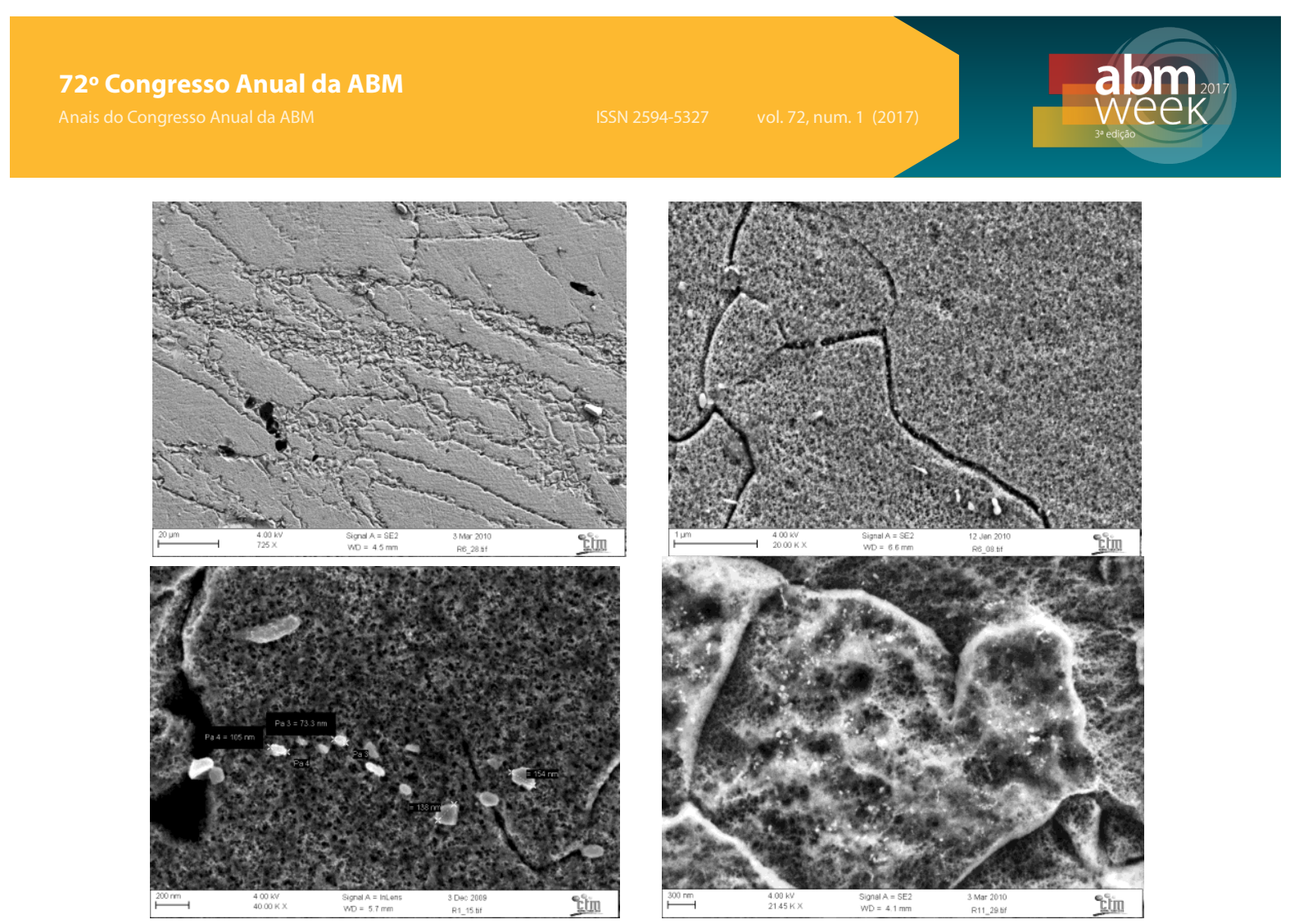

Figura 8 Microestrutura do aço ASTM F 1586: (a) Grãos MDRX incompleta; (b, c e d) Precipitados da fase Z (NbCrN) nos contornos de grãos.

O efeito da quantidade de deformação na interação recristalização precipitação a temperatura constante, mostra que o início de formação do patamar (início de precipitação) ocorre em tempos menores para maiores deformações. Veja que na condição de $1000{ }^{\circ} \mathrm{C}$ ocorre a manifestação da precipitação antes que o amaciamento alcance $50 \%$ na deformação de 0,30 com tempo de apenas $1,0 \mathrm{~s}$, Fig 5. Entretanto para uma quantidade de deformação menor de 0,15 o tempo de início de formação do patamar é de $10 \mathrm{~s}$ com amaciamento de $30 \%$. Para temperatura de $1050{ }^{\circ} \mathrm{C}$ os tempos de início de formação dos patamares são próximos de $10 \mathrm{~s}$ com um amaciamento já de $30 \%$. Para temperaturas e quantidade de deformações maiores o amaciamento estático se completa antes de iniciar a precipitação induzida.

Os ensaios de compressão a quente de duplo passe mostrou a interação entre a recristalização metadinâmica e a precipitação com a cinética de precipitação acelerada com a deformação aplicada. Outra razão da disparidade quanto ao início de precipitação nos dois tipos de ensaios deve-se a uma superestimação na determinação do tempo em escala logarítmica, devido ao método aplicado.

\section{CONCLUSÃo}

O comportamento mecânico do aço inoxidável austenítico ASTM F 1586 no intervalo de $1000-1200^{\circ} \mathrm{C}$ a $1,0 \mathrm{~s}^{-1}$ foi conduzido utilizando-se a técnica de simulação físico do processamento termomecânico por meio de testes de compressão a quente isotérmico continuo e de duplo passe de deformação em diferentes tempo inter-passe. Os principais resultados alcançados por observações mecânico-microestrutural são apresentados abaixo:

- Os ensaios de duplo passe para diferentes condições de deformação indicam que o aço ASTM F-1586 sofre recristalização metadinâmica (MDRX) em deformações maiores que 0,30 , sendo mais evidente com o aumento da temperatura e da deformação aplicada. 
- Todas as curvas de amaciamento por recristalização metadinâmica apresentaram a forma sigmoidal com expoente de Avrami (n) baixo, menor que 0,3, mostrado no aspecto microestrutural com MDRX incompleta e nas condições abaixo de $1100^{\circ} \mathrm{C}$ apresentaram formação de patamares por conta da presença de precipitados (Fase Z) induzida por deformação durante o tempo entre passe.

\section{Agradecimentos}

À Universidade CEUMA e a FAPEMA (Fundação de Amparo à Pesquisa e ao Desenvolvimento Científico e Tecnológico do Maranhão) pelo apoio financeiro.

\section{REFERÊNCIAS}

11 Palmiere, EJ. Precipitation Phenomena in Microalloyed Steels. Microalloying "95 Conference Proceedings, 1995, 307.

22 McQuenn, HJ. Development of Dynamic Recrystallization Theory. Materials Science and Engineering A, 2004; 387: 203-208.

33 Kocks, UF, Mecking, H. Physics and phenomenology of strain hardening: the FCC case. Materials Science 48, 2003, 48:171-273.

44 McQuenn, HJ. e Ryan, ND. Constitutive analyze in hot working. Materials Science and Engineering A, 2002 (322): 43-63.

55 Hench, L.; Polak, J. Third Generation Biomedical Materials. Science, 2002 (295), 1014-1017.

66 Lo, KH.; Shek, CH. e Lai JKL. Recent developments in stainless steels. Materials Science and Engineering R, 2009 (65),39-104.

77 Navarro, M.; Michiardi, A.; Castanho, O.; e Planell, JA. Biomaterials in Orthopaedics. Journal of the Royal Society Interface, 2008 (5), 1137-1158.

88 Giordani, EJ, Jorge JR, AM, Balancin, O. Proportion of recovery and recrystallization during interpass times at high temperatures on a $\mathrm{Nb}$ - and $\mathrm{N}$ bearing austenitic stainless steel biomaterial, Scripta Materialia, 2006 (55), 743746.

99 Roucoules, C, Hodgson, PD, Yue, S and Jonas, JJ. Metal. and Mater. Trans A 25A (1994) 389.

1010 Silva ES, Sousa RC, Jorge AM Jr and Balancin O. Hot deformation behavior of an $\mathrm{Nb}$ - and $\mathrm{N}$ - bearing austenitic stainless steel biomaterial. Materials Science \& Engineering: A. 2012; 543:69-75.

1111 Cipolla, L.; Danilelsen, HK. Venditti, D.; Hald, J.; Somers, M. A. J. On the role of $\mathrm{Nb}$ in Z-phase formation in a 12\% Cr steel. Scripta Materialia, 2010 (63), 324327.

1212 Wang, J. Xião, H. Determination of the Kinetics for Dynamic and Static Recrystallization by Using the Flow Curves. Materials Science Forum, 2008, (575578), 904-909.

1313 Lin, YC, Chen, MC. Study of microstructural evolution during metadynamic recrystallization in a low-alloy steel. Materials Science and Engineering A. 2009, 501 p.229-234.

1414 Sousa RC, Silva ES, Jorge AM Jr, Cabrera JM and Balancin O. Dynamic recovery and dynamic recrystallization competition on a $\mathrm{Nb}$ - and $\mathrm{N}$ - bearing austenitic stainless steel biomaterial. Materials Science \& Engineering: A. 2012; 582:96-107. 
1515 Silva, MBR; Gallego, J; Cabrera, JM, Balancin, O. e Jorge, A.M. Interaction between recrystallization and strain-induced precipitation in a high $\mathrm{Nb}$ - and $\mathrm{N}$ bearing austenitic stainless steel: Influence of the interpass time. Materials Science \& Engineering. A, Structural Materials: Properties, Microstructure and Processing, v. 637, p. 189-200, 2015. 